\title{
Structural Analysis of the Helicobacter pylori pore forming toxin, VacA
}

\author{
T. M. Pyburn ${ }^{1}$, N. J. Foegeding ${ }^{1}$., T. L. Cover ${ }^{2,3}$, and M. D. Ohi ${ }^{1,4,5}$
}

${ }^{1}$ Department of Cell and Developmental Biology, Vanderbilt University, Nashville, TN 37232

${ }^{2}$ Departments of Pathology, Microbiology and Immunology, and Medicine Vanderbilt University School of Medicine, Nashville, TN 37232

${ }^{3}$ Veterans Affairs Tennessee Valley Healthcare System, Nashville, TN 37232

${ }^{4}$ Department of Biochemistry, Vanderbilt University, Nashville, TN 37232

${ }^{5}$ Center for Structural Biology, Vanderbilt University, Nashville, TN 37232

Helicobacter pylori is a Gram-negative bacterium that colonizes the human stomach and causes gastric inflammation, leading to the development of peptic ulceration and gastric cancer in a subset of infected individuals $[1,2]$. $\mathrm{H}$. pylori infects about $50 \%$ of humans (more than 3 billion people), with a prevalence as high as $90 \%$ in some developing nations [3-5]. Gastric cancer is the third leading cause of cancerrelated deaths worldwide [6]. H. pylori-associated gastric adenocarcinoma is a rare example of a human cancer attributed to a specific bacterial infection, a connection that led the World Health Organization to classify $H$. pylori as a type 1 carcinogen.

One of the major virulence factors secreted by H. pylori is the pore-forming exotoxin VacA (vacuolating cytotoxin A), named for its ability to induce the formation of large vacuoles in eukaryotic cells [7]. VacA causes multiple cellular effects that include cell vacuolation, depolarization of membrane potential, mitochondrial dysfunction, autophagy, cell death, activation of mitogen-activated protein kinases, and inhibition of $\mathrm{T}$ cell activities [8,9]. Most VacA-induced cellular alterations require oligomerization of the toxin [10-12], insertion into the lipid bilayer to form a membrane channel [1316], and internalization into the cell [17-19]. The molecular mechanisms underlying each of these steps and the order in which they occur are not understood.

Although there is no crystal structure of the holotoxin, there is a $2.4 \AA$ three-dimensional (3D) structure of part of the p55 domain by X-ray crystallography [20] and we have characterized the 3D "snowflake"like structures of VacA oligomers at $\sim 15 \AA$ resolution by negative stain electron microscopy (EM) [21]. In order to form a functional anion-specific channel, VacA must oligomerize and insert into the lipid bilayer. However, the mechanisms by which these steps occur are not fully understood. The structural organization of membrane-bound VacA has not been characterized in any detail and the role(s) of specific VacA domains in membrane binding and insertion are unclear. Using a combination of single particle electron microscopy and biochemical techniques our work shows that membrane-bound VacA organizes into hexameric oligomers (Figure 1). Comparison of the two-dimensional averages of membrane-bound and soluble VacA hexamers generated using single particle electron microscopy reveals a structural difference in the central region of the oligomers (corresponding to the p33 domain), suggesting that membrane association triggers a structural change in the p33 domain. Analyses of the isolated p55 domain and VacA variants demonstrate that while the p55 domain can bind membranes, the p33 domain is required for membrane insertion. Surprisingly, neither VacA oligomerization nor the presence of putative transmembrane GXXXG repeats in the p33 domain is required for membrane insertion. These findings provide new insights into the process by which VacA binds and inserts into the lipid bilayer to form membrane channels. 
References:

1. Marshall, B.J. et al. Lancet, 1984. 1(8390): p. 1311-5.

2. Cover, T.L. et al. Gastroenterology, 2009. 136(6): p. 1863-73.

3. Suerbaum, S. et al. N Engl J Med, 2002. 347(15): p. 1175-86.

4. Atherton, J.C. Annu Rev Pathol, 2006. 1: p. 63-96.

5. Amieva, M.R. et al. Gastroenterology, 2008. 134(1): p. 306-23.

6. de Martel, C., et al., Global burden of cancers attributable to infections in 2008: a review and synthetic analysis. Lancet Oncol, 2012. 13(6): p. 607-15.

7. Cover, T.L. et al. Nat Rev Microbiol, 2005. 3(4): p. 320-32.

8. Gebert, B., et al. Science, 2003. 301(5636): p. 1099-102.

9. Sundrud, M.S., et al. PNAS, 2004. 101(20): p. 7727-32.

10. Vinion-Dubiel, A.D., et al. J Biol Chem, 1999. 274(53): p. 37736-42.

11. Genisset, C., et al. Infect Immun, 2006. 74(3): p. 1786-94.

12. Ivie, S.E., et al. Infect Immun, 2008. 76(7): p. 2843-51.

13. Szabo, I., et al. EMBO J, 1999. 18(20): p. 5517-27.

14. Czajkowsky, D.M., et al. PNAS, 1999. 96(5): p. 2001-6.

15. Iwamoto, H., et al. FEBS Lett, 1999. 450(1-2): p. 101-4.

16. Tombola, F., et al. Biophys J, 1999. 76(3): p. 1401-9.

17. Willhite, D.C., et al. Cell Microbiol, 2004. 6(2): p. 143-54.

18. Calore, F., et al. Cell Death Differ, 2010. 17(11): p. 1707-16.

19. Gauthier, N.C., et al. J Cell Biol, 2007. 177(2): p. 343-54.

20. Gangwer, K.A., et al. PNAS, 2007. 104(41): p. 16293-8.

21. Chambers, M.G., et al., J Mol Biol, 2013. 425(3): p. 524-35.

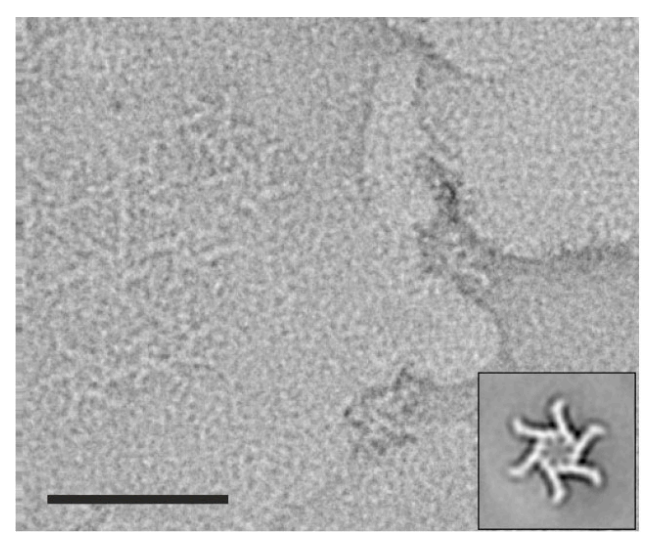

Figure 1. Negative stain EM analysis shows VacA binds membranes as hexamers. Acidified VacA was combined with vesicles and imaged using an FEI $200 \mathrm{kV}$ microscope. Class average of lipid-bound VacA shown in bottom corner. Bar, $50 \mathrm{~nm}$. Side length of inset: $42 \mathrm{~nm}$. 